Questions vives

\section{Questions Vives}

Recherches en éducation

$n^{\circ} 21 \mid 2014$

Le travail collectif des enseignants en question(s)

\title{
La division du travail éducatif : deux figures de la subjectivité en tension au sein de l'institution scolaire
}

The division of school work: two figures of subjectivity in tension within the academic institution

Louis Levasseur

\section{OpenEdition}

Journals

Édition électronique

URL : http://journals.openedition.org/questionsvives/1513

DOI : 10.4000/questionsvives.1513

ISSN : $1775-433 \mathrm{X}$

\section{Éditeur}

Université Aix-Marseille (AMU)

Édition imprimée

Date de publication : 15 septembre 2014

ISBN : 978-2-912643-45-2

ISSN : 1635-4079

Référence électronique

Louis Levasseur, "La division du travail éducatif : deux figures de la subjectivité en tension au sein de l'institution scolaire », Questions Vives [En ligne], n 21 | 2014, mis en ligne le 15 septembre 2014, consulté le 02 mai 2019. URL : http://journals.openedition.org/questionsvives/1513; DOI : 10.4000/ questionsvives.1513

Ce document a été généré automatiquement le 2 mai 2019.

\section{(†)}

Questions Vives est mis à disposition selon les termes de la licence Creative Commons Attribution Pas d'Utilisation Commerciale - Pas de Modification 4.0 International. 


\section{La division du travail éducatif : deux figures de la subjectivité en tension au sein de l'institution scolaire}

The division of school work: two figures of subjectivity in tension within the academic institution

Louis Levasseur

1 En 1997, le ministère de l'Éducation du Québec (1997) annonçait une réforme dont les milieux d'enseignement ont surtout retenu les éléments pédagogiques et particulièrement le passage des méthodes transmissives aux méthodes actives. La réforme comprend toutefois de nombreux autres volets, dont la décentralisation du système scolaire, la déconfessionnalisation des commissions scolaires, la refonte du curriculum, des réflexions de fond sur l'égalité des chances ainsi que la définition d'une triple mission de l'éducation touchant à l'instruction, la socialisation et la qualification.

2 La socialisation a donc été identifiée par le ministère de l'Éducation comme une des trois missions de l'école québécoise et nous y avons consacré l'essentiel de nos recherches au cours de la dernière décennie. Nous avons étudié la manière dont cette mission s'exerce à la fois dans les services éducatifs offerts aux élèves par le personnel technique et paratechnique (Tardif \& LeVasseur, 2010) et dans l'enseignement des différentes matières scolaires, particulièrement dans celles jouant un rôle central dans les processus de définition identitaire (individuels et collectifs) et la création du lien social comme l'histoire (LeVasseur \& Cardin, 2012, 2013; LeVasseur, Moisan \& Cardin, 2013) et la religion (LeVasseur, 2013). Si donc, l'école québécoise fait de la socialisation une de ses trois missions fondamentales, si tous les agents scolaires doivent contribuer à l'effort de socialisation des élèves, on peut se demander si tous adhèrent ou non à une même conception de la socialisation et si tous l'incarnent, ou non, dans différents dispositifs ou actions éducatives? Y a-t-il complémentarité, recouvrement, opposition dans leurs manières de travailler à la socialisation des élèves? 
Dans cet article, nous nous intéressons aux discours que tiennent les enseignants et les techniciens en éducation spécialisée (TES) sur les rapports qu'ils entretiennent aux élèves ayant des difficultés de comportement en classe et plus largement dans l'institution scolaire. Bien que la socialisation des élèves soit l'œuvre commune de tous les agents scolaires et qu'à première vue, les enseignants et les agents techniques tiennent des propos analogues sur la socialisation des élèves, l'autonomie morale de la personne, la liberté de l'enfant et la construction du sujet, les lieux institutionnels d'où ils parlent, les fonctions exercées, les problèmes auxquels font face les élèves, les particularités des milieux et les circonstances font apparaitre des différences dans les discours. Les enseignants et les TES peuvent alors ne plus adhérer aux mêmes modèles de socialisation et ne plus s'entendre quant à la priorité à donner soit à l'ordre scolaire, soit à l'autonomie du sujet, autrement dit, soit à la prééminence de la règle, soit à la liberté de l'élève, et surtout, ils peuvent commenter négativement le travail de l'autre, ce que nous illustrerons grâce à des données tirées de nos diverses recherches sur les modes de socialisation en milieu scolaire.

Dans la suite de cet article, nous montrerons que les enseignants et les TES adhèrent fondamentalement à des valeurs éducatives communes s'inscrivant dans un mouvement social et culturel qui, loin de se limiter à l'éducation, transforme les sociétés et la pensée occidentales. Ces valeurs éducatives, dans le domaine de l'éducation, tendent à faire de la personne, de l'enfant, de l'adolescent la pierre angulaire du travail des agents scolaires. Cependant, cette quasi unanimité des agents scolaires au sujet des valeurs individualistes ou subjectives que l'on peut déceler dans un ordre de discours très général orienté vers les finalités de l'éducation n'empêche pas l'existence, dans un autre ordre de discours renvoyant non plus à des principes généraux mais à des pratiques plus empiriques, de différences importantes dans la manière dont ces mêmes agents conçoivent ces mêmes valeurs. Ainsi, pour les enseignants, il n'y a de subjectivité ou de construction identitaire valable que celle qui s'inscrit dans des épreuves ou des exigences scolaires. Au contraire, pour le TES, l'élève qui se définit en résistant à la norme scolaire, ou qui, par indolence, ne s'identifie pas à l'institution, sans chercher délibérément à s'y opposer, doit être considéré, tout autant que le bon élève, comme étant capable de se construire en tant que sujet. Pour le TES, la résistance à la norme scolaire est en soi une preuve d'autonomie morale et, de ce fait, l'élève mérite inconditionnellement son empathie.

5 Il semble donc y avoir des divergences quant à la manière de définir et de construire la subjectivité de l'élève. Il peut en résulter, entre agents scolaires intervenant auprès des mêmes élèves, des mésententes, des relations tendues, de la méfiance et éventuellement des querelles et des disputes. Conséquemment, les consensus idéologiques, contrairement aux prétentions d'une certaine littérature managériale, ne sont pas nécessairement garants d'un univers institutionnel cohérent et unifié. Quant aux « dysfonctionnements institutionnels", il importe de ne pas les ramener exclusivement à des facteurs personnels ou psychologiques, mais de montrer qu'ils tiennent à des facteurs plus sociologiques ou structurels.

6 Avant de montrer l'importance que prend la subjectivation dans nos sociétés occidentales et, par la suite, la conception qu'en ont les enseignants et les TES, il importe de dire qui sont ces techniciens et dans quelles circonstances ils sont appelés à travailler en collaboration avec les enseignants. 


\section{Des enseignants et des techniciens en éducation spécialisée}

7 Avec l'entrée massive d'élèves qui ont du mal à intérioriser la norme scolaire ou qui rechignent à jouer le jeu de la scolarité, l'école doit leur porter une attention particulière, soit pour réaliser auprès d'eux un travail d'éducation, voire de médiation, soit pour les contraindre par la force au respect des règlements scolaires. Le Québec a surtout opté pour la voie de l'éducation. Au cours des années 1990, les écoles primaires et secondaires ont fait l'embauche massive de TES (Tardif \& LeVasseur, 2010). Ces techniciens exercent un travail éducatif, centré sur l'écoute et l'empathie, et encadrent les élèves qui, pour des raisons extrêmement variées, suivent des parcours scolaires incertains. Ces techniciens, contrairement aux professionnels, interviennent surtout sur la ligne de feu, dans des situations d'urgence. Leur rôle principal consiste alors à rétablir un certain ordre, non pas en imposant le règlement scolaire mais en dialoguant avec l'élève, en le calmant, puis en cherchant à assurer son bien-être, à rehausser son estime de soi. Plusieurs TES s'identifient fortement aux élèves, défendent l'idée de droit des enfants contre un monde adulte qui représenterait la force et l'autorité. Il en résulte parfois des tensions avec les enseignants qui maintiennent le cap sur la réussite scolaire, y compris pour les élèves présentant des difficultés d'apprentissage.

Cependant, nous le verrons dans la présentation de nos données empiriques, les enseignants adhèrent également à des valeurs d'écoute et d'empathie, mais plus fondamentalement, à ce que nous avons appelé une "subjectivation de la culture scolaire » (LeVasseur, 2008, 2012a), selon laquelle les savoirs trouvent leur valeur surtout en tant qu'adjuvant du développement identitaire de la personne, les savoirs et leurs assises épistémologiques s'effaçant quelque peu devant une esthétisation de la culture où l'expression de soi tend à l'emporter sur une vision strictement objective du monde (Francillon-Jacquet, 2004, p. 43).

9 Certes, la «subjectivation de la culture » que nous identifions ici à l'enseignement des matières scolaires n'est pas l'équivalente des finalités d'intégration sociale et de soutien personnel et social que nous avons définies ailleurs comme étant au cœur du travail des TES, lesquels doivent aider les élèves à rétablir un lien brisé entre ceux-ci et l'institution scolaire, pour des raisons qui ne sont pas que scolaires ou liées aux apprentissages, mais plutôt sociales, comme la distance qui existe entre certains élèves et la norme scolaire, ou psychologiques, en outre des problèmes de comportement ou encore de détresse morale (LeVasseur \& Tardif, 2005). Il n'en demeure pas moins une communauté d'esprit entre la vision de l'éducation des enseignants centrée sur le sujet, son développement intégral d'une part, et d'autre part, celle des TES axée sur la dimension socio-affective de l'élève ou encore sur une éthique du " caring " profondément ancrée dans la pensée éducative au Québec depuis les années fin 1960 (LeVasseur, 2010, 2013 ; Simard, 2013), ce que rend admirablement bien la réflexion d'une TES : «En général, les enseignants sont pas mal empathiques aussi, je pense que nous aussi on a une vision plus empathique dès le départ, mais que plus ça va, plus les enseignants ont une vision globale de l'enfant » (TES 1, école $4^{1}$ ). Or, encore une fois, bien que ces deux conceptions de l'enfant ou de l'élève puissent se recouvrir à bien des égards et trouver leur point d'origine dans une même vision moderne de l'individu en tant que valeur cardinale de l'Occident, il subsiste entre les enseignants et les TES des divergences assez fines, mais réelles, dans leur conception de la 
subjectivation, ou encore, dans les modalités de construction de la subjectivité des élèves, que nous illustrerons par des extraits d'entretiens.

10 En somme, avec la subjectivation de la culture scolaire propre aux enseignants et une subjectivation plus centrée sur une éthique du "caring», de l'écoute ou de l'empathie tout à fait caractéristique des TES, mais à laquelle adhèrent largement les enseignants, nous sommes en présence de deux figures de la subjectivité qui, dans la mesure où ces agents scolaires en conçoivent différemment les modalités de construction, peuvent être sources de tension.

\section{Subjectivation, socialisation et mouvement de la société occidentale}

11 Les recherches que nous menons sur la culture scolaire au Québec postulent que l'éducation n'est pas que le fait des pédagogues, encore moins des psychologues. Elles portent, en effet, sur les missions d'instruction et de socialisation de l'école québécoise (MÉQ, 1997) et montrent que l'enseignement des matières scolaires et les services d'encadrement aux élèves s'inscrivent dans un vaste mouvement de modernisation des sociétés occidentales ou dans la perspective d'une mutation des modes de socialisation qui oriente fortement le travail des agents scolaires. Cette idée est capitale pour notre propos. Nous faisons effectivement l'hypothèse que malgré l'éventuelle convergence entre les enseignants et les TES au sujet de l'individu comme valeur fondamentale de l'éducation, subsiste des tensions dans leurs motifs et modalités d'intervention respectifs auprès des élèves qui dérogent au règlement scolaire. Ces tensions sont certes attribuables à des facteurs psychologiques ou à une absence de sympathie mutuelle entre les agents scolaires, mais notre regard se porte plutôt sur des processus sociaux et culturels qui déterminent le fonctionnement des institutions sociales, y compris l'école et le travail que les agents scolaires y effectuent avec les élèves. Notre but est de montrer qu'avant de construire un ordre scolaire, une société, une cité, une civilisation, avant de travailler à la consolidation de l'intérêt commun ou même de transmettre des savoirs généraux à dimension "universelle», les agents scolaires du Québec travaillent à la production de sujets individuels et, en cela, suivent le mouvement de la modernité (LeVasseur, 2012a, 2012b; LeVasseur \& Cardin, 2012). Autrement dit, leurs actions et pensées s'inscrivent dans des structures qui les englobent et qui orientent la conception qu'ils se font de leur travail et des modalités d'intervention auprès des élèves ayant des difficultés de comportement. Deux questions surgissent alors. En quoi consiste ce fond commun d'idées qui étaye l'action de ces agents scolaires? Pourquoi l'adhésion fondamentale des agents scolaires aux valeurs individualistes de la modernité n'empêche pas, dans plusieurs cas, l'absence de coordination entre les actions qu'ils mènent auprès des élèves récalcitrants?

12 La culture occidentale se caractérise par le soupçon quant aux discours de légitimation des grandes instances, qu'elles soient institutionnelles, morales, religieuses ou même scientifiques. Nous vivons dans des sociétés où la critique est passée dans les mœurs et devient en quelque sorte une compétence de tous les individus (Boltanski, 1990). L'instance de légitimation du discours possible sur le monde passe par le sujet lui-même, lequel ne se laisse plus enfermer dans une tradition, dans un impératif moral, un dogme religieux, une idéologie politique ou encore dans un ensemble de rôles sociaux auxquels il 
se conformerait passivement (Beck, 2001). La réflexivité devient d'ailleurs une pratique s'inscrivant au cœur de la construction identitaire des sujets (Martuccelli \& de Singly, 2009). La sociologie tend ainsi à montrer qu'actuellement l'acteur social ne fait pas qu'intérioriser passivement un ensemble de valeurs qui donneraient sa cohésion au tout social, mais qu'il construit sa propre expérience en tissant des liens entre des registres qui relèvent de logiques d'action sociales en tension auxquelles il donne une certaine unité (Dubet, 1994).

13 Si les rapports entre l'individu et la société tendent à changer en faveur d'une prééminence accordée au sujet, l'affaiblissement du modèle institutionnel identifié par Dubet montre également que de nouveaux espaces d'interprétation s'ouvrent pour le sujet. Le fonctionnement des institutions n'est pas étranger à l'idée d'une production d'un sujet autonome. Le programme institutionnel (Dubet, 2002) consiste en un mode de socialisation, ayant prédominé jusque dans les années 1960 ou 1970, selon lequel les valeurs transmises par l'institution définissent les rôles qu'assument les individus (notamment le rôle d'élève), modèlent leurs conduites et marquent leur personnalité (Dubet \& Martuccelli, 1996a, 1996b). Un tel rapport social se définit par la correspondance entre l'institution et l'individu au point où celui-ci recevrait de celle-là une partie de son identité. Or, si l'institution scolaire ne se caractérise plus par son haut degré d'intégration, par sa force régulatrice, par son pouvoir d'enrôlement, par sa capacité à transmettre des valeurs qu'intériorisent les agents sociaux comme les élèves à l'école, il revient à ceux-ci de définir le lien qu'ils établissent à l'institution, aux études, à la culture scolaire. Il leur revient de donner un sens à leur expérience dans les cadres d'une scolarité qui n'est plus définie a priori et de manière définitive. Le sujet devient en quelque sorte l'auteur du récit de sa propre scolarité. Or, tant les enseignants que les TES, dans leur travail respectif et dans leurs modalités d'intervention avec les élèves, adhèrent à de telles valeurs de subjectivation.

14 Toutefois, nous soutenons qu'il y a d'importantes divergences entre les enseignants et les TES quant à la manière dont la construction de la subjectivité des élèves ayant des difficultés de comportement doit se réaliser, ce qui expliquerait le fait que dans la réalité concrète de leurs interventions avec ceux-ci, ils ne parviennent pas toujours à arrimer leurs actions. Avant d'illustrer ces divergences, donnons sommairement des indications méthodologiques sur les recherches dont sont issues nos analyses.

\section{Indications méthodologiques}

15 Le présent article repose sur des données tirées de deux principales recherches. La première porte sur la division du travail éducatif menée au cours de la décennie 2000 . Nous avons visité 4 établissements de la région du Grand Montréal, deux appartenant à l'ordre d'enseignement primaire et deux à l'ordre d'enseignement secondaire, afin d'y suivre le travail qu'y effectuent les TES qui, nous l'avons vu, ont pour mandat d'encadrer les élèves qui entretiennent un rapport d'extériorité par rapport à la norme scolaire. Trois de ces établissements accueillent surtout des élèves des milieux défavorisés et des classes moyennes, les élèves issus des milieux favorisés étant de plus en plus scolarisés dans des établissements privés qui sélectionnent les élèves, et le quatrième établissement accueille des élèves d'un milieu socioéconomique très défavorisé. Nous sommes demeurés environ deux semaines dans chaque établissement et nous avons réalisé une quarantaine d'entrevues semi-dirigées s'échelonnant en tout sur une trentaine d'heures avec des TES, 
des enseignants et d'autres agents scolaires travaillant avec les TES, notamment des chefs d'établissement, des psychoéducateurs, des techniciens en toxicomanie, des policiers et même des élèves. Le but de la recherche consistait à déterminer les fonctions assumées par les TES et à décrire les rapports existant entre eux et les autres agents scolaires.

Dans le cadre de la seconde recherche portant sur la mission de socialisation de l'école québécoise vue à travers l'enseignement, nous avons rencontré 34 enseignants de toutes les matières scolaires en 2007, 19 enseignants de religion en 2008 et 22 enseignants d'histoire en $2009^{2}$. Tous enseignaient dans des établissements de l'ordre d'enseignement secondaire. Le principal objectif des entrevues semi-structurées consistait à déterminer comment les enseignants estimaient tenir compte du lien social par le biais des finalités éducatives, des contenus d'enseignement et des dispositifs pédagogiques.

Quels liens faut-il donc établir entre ces deux recherches? Nous nous sommes longtemps interrogé, dans le cadre de notre première recherche sur la division du travail éducatif, sur le fait qu'il puisse subsister des tensions entre des agents scolaires partageant de mêmes conceptions axiologiques au sujet d'une dimension de leur travail ${ }^{3}$. Au départ, nos analyses ont porté sur le personnel technique et paratechnique. Nous en avons fait le portrait statistique, identifié les fonctions qu'ils exercent et décrit les rapports sociaux qu'ils entretiennent avec les enseignants qu'ils doivent appuyer dans la gestion des élèves au comportement difficile (Tardif \& LeVasseur, 2010). Cependant, tout au cours de ces analyses, nous avons cherché à identifier les conditions favorables à la collaboration entre les enseignants et les techniciens. Pour que des agents scolaires aux statuts professionnels et aux fonctions différents s'entendent, faut-il qu'ils partagent une même vision de l'éducation, qu'ils occupent des territoires à la fois différents mais complémentaires, que leurs fonctions se juxtaposent ou qu'elles se recouvrent? Les tensions qui surviennent entre les enseignants et les TES sont-elles de tâches, de rôle, de représentations, de pouvoir hiérarchique, de statuts professionnels, d'identités professionnelles? Sont-elles reliées à la différence de leur formation? Or, la seconde recherche portant sur les matières scolaires nous offre des données qui, croisées avec celles de la recherche sur la division du travail éducatif, permettent de jeter un éclairage particulier sur le fait que l'adhésion à de mêmes valeurs éducatives puisse aller de pair avec des pratiques divergentes et des tensions entre agents scolaires.

Dans ce qui suit, nous insisterons donc sur le fait que des enseignants et des TES tiennent un discours analogue au sujet des élèves au comportement difficile, mais qui comprend néanmoins des divergences, lesquelles peuvent conduire à des manières différentes d'intervenir auprès de mêmes élèves, ce qui entraîne des mésententes et même de la rancœur chez les TES qui doivent se soumettre hiérarchiquement au pouvoir des enseignants. Les justifications que les uns et les autres donnent de leurs actions font alors voir que la gestion la plus routinière, ou qui semble aller de soi, se caractérise souvent par une absence de coordination, et ce, tout autant à l'ordre d'enseignement primaire que secondaire.

\section{Les enseignants et la subjectivation de la culture scolaire}

19 La culture de la subjectivation que nous avons présentée précédemment (partie 2) a une influence considérable sur les programmes d'enseignement et sur les représentations que 
les enseignants du secondaire se font de leur propre discipline et de la fonction sociale de l'école (LeVasseur, 2008, 2010) ${ }^{4}$. Cette fonction sociale ne consiste plus en une intériorisation passive des normes scolaires ou encore de récits normatifs ou collectifs caractéristiques de l'enseignement de la religion et de l'histoire, mais plutôt en le développement de la capacité réflexive de l'élève, celle de prendre distance par rapport aux discours ambiants et de définir, par lui-même, sa propre représentation des choses, du monde, des rapports qu'il entretient aux autres, etc. Nous serions donc passés d'une socialisation par intériorisation à une socialisation par distanciation, processus que Dubet associe à une logique de subjectivation (Dubet \& Martuccelli, 1996a, 1996b), et c'est ce que nous illustrerons par des extraits d'entretiens menés avec des enseignants (partie 4.1.). Les enseignants ne semblent pas changer de discours lorsqu'il est question d'élèves présentant un comportement plus distant par rapport à la norme scolaire, d'élèves plus indolents ou même aux prises avec des problèmes d'ordre cognitif, psychologique ou social. Ils insistent sur le fait que les élèves représentent la valeur centrale de leur travail, que l'institution doit se mettre à leur écoute et qu'une partie fondamentale de leur travail consiste à établir un lien significatif avec les élèves ayant de la difficulté à fonctionner dans le cadre scolaire (partie 4.2). Toutefois, lorsqu'ils commentent le travail des TES avec ce même type d'élèves, ils se réclament alors d'une logique non plus socio-affective mais institutionnelle selon laquelle les élèves doivent se conformer à un ordre scolaire préexistant (partie 4.3.). Dans ce qui suit, nous présenterons le discours des enseignants (partie 4), puis celui des TES, lesquels fustigent littéralement les enseignants qui s'en remettent, de près ou de loin, à toute forme de référence institutionnelle ou bureaucratique de l'éducation (partie 5).

\subsection{Subjectivation et enseignement des disciplines scolaires}

20 La très grande majorité des enseignants que nous avons rencontrée au cours de nos recherches sur la socialisation en milieux scolaires adhèrent à l'idée d'un individu autonome sur les plans cognitif, moral et esthétique. Qu'entendons-nous par une telle autonomie ? Le fait que l'élève est invité, par l'enseignant, à se positionner de manière critique par rapport à l'enseignement, aux contenus transmis et au savoir disciplinaire. Aucun enseignant n'a déclaré, au cours de nos recherches, que l'élève pouvait se passer de l'enseignement des matières scolaires dans sa construction identitaire et dans l'édification de sa représentation du monde. Mais presque tous ont exprimé, explicitement ou tacitement, l'idée selon laquelle il revenait à l'élève de justifier son adhésion ou sa non adhésion à telle ou telle vision de l'histoire, de la religion, de la littérature et même de la science.

21 Par exemple, l'analyse de nos données montre essentiellement qu'un enseignement axé sur la transmission d'un récit collectif unitaire et hérité ne mobilise plus les enseignants d'histoire de Montréal. En fait, à la transmission de ce récit historique centré sur les origines des Canadiens français ou des Québécois d'origine canadienne-française ${ }^{5}$, sur les événements catalyseurs de vie collective, sur les personnages historiques susceptibles de galvaniser la nation et de cimenter l'identité collective se substitue la production d'un sujet autonome sur le plan de la pensée :

Notre appartenance c'est d'abord notre choix, puis, aussi, c'est multi, c'est varié ce n'est pas quelque chose qui est dicté pour vous, c'est un choix que vous faites. Mon sens du nationalisme est ancré dans James Joyce. Pour être un artiste ou un vrai individu, tu laisses les nations, les religions puis la langue derrière toi pour te 
découvrir. Qui suis-je ? Et ça c'est quelque chose que j'enseigne, évidemment. (Sujet 7, histoire $\left.{ }^{6}\right)$

La fonction ultime est de faire des individus libres, des libres penseurs, des « nonmanageables ", des gens qu'on ne pourra pas manipuler par une idéologie quelle qu'elle soit. Ma force, c'est de donner tous les repères pour ça, et je ne vois pas l'histoire comme un instrument, par exemple, pour mouler un élève d'origine étrangère. L'idée, c'est de lui donner des repères. (Sujet 2, histoire)

Il en va de même des enseignants de religion. L'idée d'une forte subjectivation du rapport à la religion est décelable dans à peu près tous les propos des enseignants, qu'ils soient de Montréal ou de Québec, en début ou en fin de carrière, y compris ceux qui enseignent dans les écoles privées dont certaines peuvent conserver un lien avec le projet d'une école confessionnelle. Les enseignants disent alors insister moins sur la dimension religieuse de la religion, donc pourrions-nous dire ici, sur l'éveil de la foi et de la croyance dans un cadre orthodoxe, prescriptif et institutionnalisé, que sur la dimension éthique ou spirituelle de la croyance, ce qui renvoie à l'idée d'une certaine indépendance du croyant face à la confession religieuse et à son orthodoxie. À la limite, se décèle, dans les propos des enseignants, la volonté de favoriser un rapport non plus collectif mais subjectif à la croyance. La croyance n'est plus communautaire mais individuelle. À la question de savoir si l'enseignement va dans le sens de la formation d'une identité collective (au sens de communauté de croyants) ou d'identités individuelles, voici certaines réponses d'enseignants presque choisies au hasard, tant cette tendance à individualiser le rapport à la foi est récurrente dans le discours de tous les enseignants :

L'enseignement, ce n'est pas de l'évangélisation. Il y a une distance qui laisse à

l'élève sa liberté de penser. (Sujet 2, religion)

Le but c'est de faire en sorte qu'ils réfléchissent, qu'ils fassent des choix, qu'ils posent des gestes qui sont en accord avec ce qu'ils croient réellement. (Sujet 9, religion)

Le prof en avant, il n'est pas censé avoir les réponses. S'il a toutes les réponses, ce n'est plus de l'esprit critique. Ce qu'il peut faire développer, c'est une espèce d'autonomie dans la façon de réfléchir, c'est de mettre de côté les émotions et de dire « voici, on va essayer de comprendre et ton opinion, tu la garderas, mais au moins tu seras capable de la nuancer, de la rationaliser. » (Sujet 10, religion)

Si toi, tu n'acceptes pas le discours que tes parents t'ont donné par rapport à la religion, comment tu vis ta vie maintenant ? Et là bien souvent il se disent : "J'en ai pas de balises ; je ne sais pas vers où aller ». C'est quoi le sens de ta vie maintenant? La spiritualité pour moi, c'est le contact avec soi-même. Donc moi, je veux qu'ils puissent définir eux-mêmes leur spiritualité, leur sens de la vie. Qu'on rejette ou qu'on accepte les croyances de certaines religions, pour moi ça, ça t'appartient. Mais on les a vues les croyances, on a vu sur quoi se base chacune des religions. Et maintenant, qu'est-ce que t'en fais? Qu'est-ce que t'en penses? Pourquoi tu contestes ça ? (Sujet 17, religion)

Dans ces extraits d'entretien que nous venons de citer, la question de la formation de l'individu l'emporte de manière décisive sur celle de la transmission d'un cadre normatif qui préexisterait à l'individu et qu'il suffirait à celui-ci d'intérioriser afin de se définir une identité personnelle ou d'affiner sa vision du monde. À la limite, ce qu'il y a de commun dans nos sociétés et dans le discours de la très grande majorité des agents scolaires que nous avons rencontrés, c'est la priorité reconnue à chacun de vivre selon ses propres convictions et de définir ou même d'affirmer ce qui le rend irréductible aux autres sur le plan identitaire (Taylor, 1992). Cette prééminence de l'individu et de sa liberté se perdelle lorsqu'il est question de l'édification d'un ordre scolaire? Les enseignants adhèrent- 
ils toujours au respect de la subjectivité des élèves lorsque l'expression de celle-ci menace l'ordre établi dans la classe ou l'établissement?

\subsection{Subjectivation et éthique du " caring »}

des difficultés de comportement ? La prédominance que les enseignants reconnaissent au sujet (ou à l'élève) par rapport aux grands référents normatifs de la société, que ceux-ci soient inscrits dans les grandes religions, les idéologies politiques ou les grands récits (Lyotard, 1979), ou même par rapport aux traditions disciplinaires, peut-elle ne pas trouver son pendant dans la gestion que les enseignants font des élèves au comportement difficile ? L'élève qui est encouragé à affirmer son indépendance d'esprit face aux grands référents normatifs perd-il sa liberté face à un ordre scolaire que l'enseignant cherche à maintenir ou au règlement scolaire qu'il convient de respecter ? L'éthique du "caring ", de l'écoute, de l'empathie cède-t-elle le pas à une conception de l'éducation plus universelle, plus rationnelle, plus intellectuelle? Ou encore, à une conception de l'ordre scolaire plus impersonnelle, plus centrée sur l'impératif du bien commun et l'observation objective de la règle conduisant à une mise entre parenthèses du sujet? Dans la mesure où les enseignants tiennent un discours très général, un discours de principe sur l'éducation, il semblerait que non. Écoutons ce que les enseignants disent à ce sujet ${ }^{7}$ :

Il faut être capable de motiver les élèves, de leur donner de l'affection afin de les aider dans les travaux... Mais être capable de leur donner de l'affection, se mettre contre l'enfant, lui parler, jaser, être près de l'enfant. On dirait que les enfants, ici, nous font des crises parce qu'ils cherchent l'adulte près d'eux et qu'ils se sentent insécurisés. (Enseignante 1, école 1, primaire)

Je pense que d'abord, pour travailler avec ces jeunes-là, il faut que tu les acceptes comme ils sont, que tu aies cette relation dont je parle, parce que si tu ne l'as pas, c'est très difficile de les faire avancer et de leur faire aimer l'école. (...) Qu'ils soient heureux. Puis qu'ils soient heureux... Tant mieux s'ils sont heureux. On est en milieu scolaire... qu'ils soient heureux à l'école. (Enseignante 1, école 2, secondaire) Je pense que l'enfant, il faut s'assurer qu'il soit en bien-être, qu'il soit heureux d'être à l'école, et en ayant ça éventuellement ça va les amener vers les apprentissages et la réussite. Puis oui, que cet enfant-là soit bien à l'école au départ, après il va être apte à l'ouverture, éventuellement il va en faire des apprentissages, puis il va finir par réussir, si tout d'abord il est bien. (Enseignante 2, école 4, primaire)

Quand on est enseignant, ce qu'on veut, c'est leur apprendre quelque chose, sauf que, même en enseignant, des fois on les éduque. Puis je pense que c'est une relation qui est quand même importante et qui les rapproche de nous. Ils se rendent compte qu'on est là pour eux, pas juste pour leur enseigner, mais aussi pour les aider s'ils ont des problèmes, à régler leurs conflits. Nos élèves savent très bien que s'ils ont un problème, ils peuvent venir nous voir à n'importe quel moment, que ce soit pendant une pause, avant ou après l'école ou à l'heure du dîner. (Enseignante 1, école 3, secondaire)

L'enseignante précédente dit valoriser la gestion de classe et les interactions avec les élèves, surtout si elles permettent de rétablir une relation difficile avec un élève. Il est même souhaitable que surviennent, dans le cours de l'enseignement, des conflits avec les élèves de manière à ce qu'elle puisse consolider son « autorité ». En cela, l'enseignante se trouve à avaliser l'idée d'un chahut traditionnel qui renforcerait l'ordre scolaire et qui est à distinguer d'un chahut plus anomique et général qui apparaît avec l'école de masse (Testanière, 1967). Conséquemment, de ce point de vue, les écarts de conduite des élèves 
offrent la possibilité d'une pleine réalisation professionnelle de l'enseignante. L'écoute et l'empathie sont alors à concevoir comme des ressources dont elle peut bénéficier. Nous verrons cependant que les enseignants ne sont pas toujours enclins à faire preuve d'empathie, surtout lorsqu'il leur devient évident qu'il y a une limite à ne pas franchir dans ce registre (partie 4.3.) et que cette ressource dont ils se servent avec profit constitue également une ressource dont se servent les TES (cf. partie 5).

\subsection{Les réticences des enseignants face au « caring »}

Dans l'absolu, les enseignants semblent donner la priorité, en toutes circonstances, à la personne de l'élève et à son écoute. Cependant, les enseignants, y compris certains dont nous venons de citer le discours, nuancent très vite leur propos dès qu'il a été question, en cours d'entrevue, des apprentissages qui relèvent de leur propre travail mais dont les TES n'ont pas à se soucier. Voici quelques extraits où les enseignants se réclament des apprentissages et des savoirs afin de marquer leur opposition à une écoute désintéressée à laquelle s'identifient les TES et qui, pour les enseignants, demeure ni possible, ni souhaitable. L'enseignante suivante, dont nous venons de citer les propos, a débuté son discours en priorisant l'écoute et les liens affectifs devant être établis avec l'élève, puis, dès qu'il a été question des TES, a cherché à se démarquer et à montrer que l'écoute devait s'arrimer à une vision plus globale de l'éducation incorporant les apprentissages :

Quand on dit que c'est pas grave les évaluations, les apprentissages, que c'est le bien-être de l'élève qui est important, je suis d'accord, mais pas à n'importe quel prix. Je pense qu'on peut montrer que oui, ils sont dans une école régulière où il y a quand même une base à respecter. C'est plate à dire mais on a quand même des comptes à rendre. Un enfant qui est bien à l'école mais qui ne travaille pas, qui ne fait rien, ça va un peu à l'encontre de... Il y a quand même un minimum de choses à respecter. Je ne suis pas rendue au point de dire que ce n'est pas important l'évaluation et les apprentissages. Mais oui, qu'ils se sentent bien et qu'ils aient le goût de venir à l'école, je pense que c'est déjà beaucoup. (Enseignante 2, école 4, primaire)

27 La démarcation entre les mondes de l'enseignement et de l'affectif n'est pas toujours nette. Les enseignants doivent continuellement pondérer leurs interventions en fonction de l'un ou de l'autre. L'harmonie entre les deux semble extrêmement difficile à réaliser, alors que le TES, lui, n'aurait qu'à se soucier de l'écoute :

Pour l'enseignant, le règlement c'est ça ou c'est pas ça, c'est pas les deux à la fois, et puis l'enseignant aussi c'est la matière qu'il enseigne, c'est ça d'abord son rôle, c'est d'enseigner. Mais l'éducateur (TES) qui n'enseigne pas est capable, lui, d'aller chercher l'élève, d'établir une certaine relation plutôt personnelle. Donc je trouve que ces personnes-là visent plus le côté affectif, alors que nous, enseignants, on vise plus le côté académique. Et c'est ça qui pose un petit peu problème. (Enseignante 2, école 2, secondaire)

Ce "qui pose un petit peu problème", pour reprendre les mots de l'enseignante précédente, touche à la difficulté de passer du registre des apprentissages à celui de l'affectif et de l'écoute, surtout dans le cas des élèves résistant à la norme scolaire. Cependant, même lorsque les enseignants cherchent à se démarquer des TES afin de mieux valoriser la dimension de leur travail et de leur identité professionnelle qui touche aux apprentissages, ils insistent pour ne jamais abandonner totalement l'idée que l'on « enseigne à des personnes » : 
Bien c'est enseigner, d'abord, moi, je dois leur transmettre des connaissances. C'est sûr qu'il y a la dimension humaine qui vient avec ça. Je n'enseigne pas à des robots, j'enseigne à des personnes qui vivent des choses. (Enseignante 2, école 3, secondaire) des réticences face aux TES qui ne font pas avancer les élèves dans leurs travaux. Elle aussi se trouve à souligner l'importance des apprentissages en réaction au travail effectué par le TES :

C'est parce que quand l'enfant est sorti, le travail ne se fait pas. Alors l'éducateur spécialisé (TES) passe son temps à, mettons, faire des messages d'explication, jaser avec les enfants, pourquoi il fait ceci, pourquoi il fait cela. Quand ils arrivent en classe, les travaux ne sont pas faits, ils sont obligés de les faire, alors ça les met en furie parce qu'on les fait travailler, ils n'ont pas le droit de jouer. Parce qu'ils sont en retard, on leur fait continuer leur travail... (Enseignante 1, école 1, primaire)

On a parfois l'impression que les enseignants inscrivent leur discours dans un double registre. Sachant que des TES misent sur l'empathie pour entrer en relation avec un élève, certains enseignants discréditeront l'empathie, après en avoir vanté les vertus éducatives, s'ils sentent que l'élève en question échappe à leur influence pour accorder sa confiance au TES. En revanche, le TES aura d'autant plus d'empathie envers un élève qu'il le sait délaissé par les enseignants. Le TES croit réussir professionnellement dans la mesure où il persiste à maintenir un lien avec un élève que les enseignants, après avoir essayé, renoncent à établir.

En somme, contrairement aux TES, les enseignants maintiennent l'idée d'une réussite scolaire devant passer par les apprentissages. Quant aux TES, nous le verrons, ils tiennent un discours qui recouvre celui des enseignants en ce qu'ils valorisent, et encore plus fortement que ne le font les enseignants, les relations interpersonnelles avec les élèves. Cependant, ils sont les représentants d'un univers domestique où la chaleur et la proximité des rapports doit l'emporter sur toute autre dimension de l'éducation (Derouet, 1992), et ils entretiennent des doutes quant à la réelle capacité des enseignants à faire preuve d'empathie envers l'élève, dans la mesure où la vision de l'éducation de ceux-ci comprend l'idée d'une réussite et d'un ordre scolaires devant s'imposer à l'élève.

\section{Les techniciens en éducation spécialisée et la subjectivation socio-affective inconditionnelle de l'élève}

Notre recherche sur la division du travail éducatif ne porte pas sur la " culture scolaire ", mais en partie sur la socialisation ou ce que nous avons appelé le travail éducatif du personnel d'encadrement technique et paratechnique des écoles québécoises. Elle a conduit au constat que la mission de socialisation, dans certains milieux, a désormais plus de poids que la mission d'instruction, et que tout comme pour les enseignants, cette socialisation est fortement centrée sur l'idée de subjectivation (LeVasseur \& Tardif, 2005), mais d'une subjectivation qui repose inconditionnellement sur le respect et le soutien affectif, moral et social de la personne. On retrouve même chez les TES une présomption selon laquelle ils savent écouter de manière attentive et authentique les élèves, alors que les enseignants se cantonneraient "par la force des choses" dans une logique académique et administrative : 
L'éducateur c'est pas un luxe, c'est quelque chose qui permet souvent (aux élèves) d'être écoutés là où ils ne sont pas entendus... Mais des fois, ils ont vraiment besoin qu'on prenne le temps de s'arrêter et d'écouter ce qu'ils ont à dire. Et ça, les enseignants n'ont pas le temps par la force des choses. Des fois ils le font... En tout cas, il y a un manque là. (TES 1 , école 1 , primaire) face à des élèves que l'on pourrait qualifier de récidivistes. Après plusieurs offenses et plusieurs tentatives de réparation de la situation avec un élève, l'enseignant finit par perdre patience, sinon confiance. Il abandonne alors sa fonction d'écoute, s'en remet au règlement scolaire et se replie sur l'enseignement :

L'enseignant a un groupe à faire réussir, il a de la matière à transmettre ; le reste de la classe doit passer, la matière faut qu'elle se donne. Puis je lui demande (à l'enseignant): «as-tu cinq minutes pour essayer de comprendre pourquoi il (l'élève) a réagi comme ça ?» - «Là, je commence à ne plus en avoir des cinq minutes (enseignant) » rendu à la troisième étape comprends-tu? "J'en ai donné des cinq minutes, je commence à ne plus en avoir (enseignant) ». (TES 1, école 3, secondaire)

Non seulement l'écoute serait-elle compromise pour des raisons touchant au trop grand nombre d'élèves dans les classes, au manque de temps, à l'absence de ressources, au fait qu'il faille faire progresser les élèves sur le plan des apprentissages, mais des TES estiment que trop d'enseignants afficheraient carrément de l'insensibilité par rapport aux élèves. En revanche, la sensibilité aux difficultés des élèves devient ce que les TES estiment être leur force :

Il faut être à l'écoute des élèves. Si tu t'en vas enseigner en disant, bon moi j'ai de la matière à passer puis just too bad, le reste j'en fais pas, ben le résultat c'est ça. Avec les jeunes d'aujourd'hui, faut s'impliquer, faut les écouter, faut les comprendre, faut se mettre dans leur peau. C'est pas toujours des choses faciles avec les familles reconstituées qu'on a et puis les familles monoparentales... J'ai l'impression souvent que les enseignants ont eu la vie facile, eux, et qu'ils ne peuvent pas comprendre c'est quoi d'avoir la vie dure. Et puis les enseignants qui sont plus à l'écoute des élèves, c'est les enseignants qui ont eu la vie dure. (TES 1, école 2, secondaire)

Et je pense intervenir dans un cadre où justement je suis beaucoup en opposition à la fermeté et... je vais employer des mots un peu plus forts, mais c'est pas grave. Mais pour moi, c'est quasiment de l'obscurantisme, de l'inquisition. On rentre dans la vie des jeunes, on impose des formules, on impose des idées, on importe notre credo, notre façon de faire, puis on n'écoute pas l'hérétique, on va le brûler avant de l'écouter. C'est une aberration. (TES 1, école 1, primaire)

Les quelques extraits que nous venons de citer suffisent à donner le ton, à situer la position des TES par rapport aux enseignants. L'enseignant écoute l'élève, lui enseigne, est au centre de l'institution scolaire, a l'oreille de la direction, travaille de concert avec les autres professionnels dans l'école, mais le TES, n'étant pas un professionnel, lui est subordonné. Certains TES en conçoivent presqu'une forme de ressentiment qui vient radicaliser leur empathie, leur sollicitude, leur engagement moral et affectif dans leurs relations avec les élèves. Et ce ressentiment ne peut que grandir lorsque des enseignants prennent l'initiative, unilatéralement, de "défaire " leurs interventions ${ }^{8}$. Le TES se retrouve dans une situation où, n'ayant pas de formation professionnelle ni pour "guérir " ni pour diagnostiquer l'élève, il ne peut que l'encadrer, faire preuve de sollicitude envers lui, l'écouter. D'où sa circonspection envers toute forme de ressource autre que celle de l'empathie ou de l'écoute et pour toute forme institutionnelle qui porte un jugement, qui mesure, qui qualifie, qui classe, qui évalue, qui réduit l'élève à un 
principe d'équivalence. D'où, également, la tension entre les TES et les enseignants, lesquels, nous l'avons vu, insistent sur le fait que leur travail ne peut pas consister exclusivement en une écoute bienveillante de l'élève ; car il implique de le faire réussir sur le plan scolaire.

\section{Conclusion} comme donnée de soi, sans épreuve. Personne n'est plus grand qu'un autre dans le registre de l'amour ou, plus précisément, ici, de l'agapè que Boltanski (1988) définit comme un état dans lequel tout individu est grand du seul fait d'être, alors que les enseignants, eux, établissent la grandeur des élèves, formulent des attentes par rapport à eux, sont à la limite dans une relation de don et de contre-don qui suppose que l'autre nous rendra ce que l'on a pu lui donner. En clair, l'enseignant refuse, au-delà d'un certain seuil, de donner de son temps, de son énergie, de sa volonté, de sa vitalité et de faire profiter de ses compétences à un élève qui lui demeure fermé ou réfractaire et qui n'accepte pas de lui rendre un engagement dans les études équivalent au don que l'enseignant fait de lui-même pour l'aider. Le TES croit pouvoir franchir ce seuil et parvenir de manière tout à fait désintéressée à entrer en relation avec l'élève.

Pour l'enseignant, il existe un étalon de mesure arrimé à la réussite et à la performance, mais également à la capacité de l'élève de se construire soi-même. Mais l'élève qui refuse de se construire en prenant appui sur l'institution scolaire, celui-là sera rejeté par l'enseignant, mais non par le TES. D'où, par rapport à l'enseignant, la grandeur présumée du TES qui réside dans une acceptation de l'enfant permettant de le percer à jour, alors que l'enseignant pose comme condition à l'acceptation de l'enfant que celui-ci puisse se construire sur le plan identitaire ou subjectif en prenant appui sur l'institution dont l'enseignant est le représentant.

En somme, les enseignants cherchent continuellement, dans leur discours, à mettre en avant une écoute qui bénéficierait aux apprentissages, alors que les TES s'en tiennent à une écoute qui, à la limite, pourrait se suffire à elle-même, une écoute plus désincarnée, plus éthérée ne devant pas se transmuer, pour les élèves ayant des difficultés, en condition sine qua non de la réussite. Les enseignants entretiendraient donc un rapport plus instrumental à l'écoute, comme s'ils attendaient un «retour sur leur investissement ", ce que refusent catégoriquement bon nombre de TES.

Nous sommes donc en présence de deux figures de la subjectivité qui se recouvrent largement, mais qui comportent néanmoins des divergences, surtout décelables dans la manière dont les enseignants et les TES aident les élèves à construire leur propre subjectivité. En effet, les enseignants conçoivent la subjectivité comme s'incarnant dans des dispositifs scolaires, tandis que les TES la conçoivent prioritairement comme étant fondée sur une relation d'aide, laquelle, pour se réaliser, peut se passer des épreuves et de l'institution scolaires dont les enseignants sont les représentants. À la limite, les réflexions de certains TES donnent à penser qu'ils se perçoivent comme étant plus nécessaires à la réalisation de soi de l'élève ayant des difficultés que les enseignants euxmêmes. 


\section{BIBLIOGRAPHIE}

Beck, U. (2001). La société du risque. Sur la voie d'une autre modernité. Paris : Éditions Flammarion.

Boltanski, L. (1988). L'amour et la justice comme compétences. Paris : Éditions Métailié.

Boltanski, L. (1990). Sociologie critique et sociologie de la critique. Politix. Revue des sciences sociales du politique, 3(10), 124-134.

Derouet, J.-L. (1992). École et Justice. Paris : Éditions Métailié.

Dubet, F. (1994). Sociologie de l'expérience. Paris : Éditions du Seuil.

Dubet, F. (2002). Le déclin de l'institution. Paris : Éditions du Seuil.

Dubet, F., \& Martuccelli D. (1996a). Sociologie de l'expérience scolaire. Paris : Éditions du Seuil.

Dubet, F., \& D. Martuccelli (1996b), Théories de la socialisation et définitions sociologiques de l'école. Revue française de sociologie, 37(4), 511-535.

Francillon-Jacquet, F. (2004). L'enfant comme personne : un fondement culturel de l'Éducation nouvelle. In A. Ohayon, D. Ottavi \& A. Savoye (dir.), L'Éducation nouvelle, histoire, présence et devenir (pp. 29-45). Berne : Peter Lang.

LeVasseur, L. (2007-2008). Bourse de développement de la recherche de la Faculté des sciences de l'éducation de l'Université Laval.

LeVasseur, L. (2007-2010). La contribution des matières scolaires à la constitution de la culture commune , Conseil de recherche des sciences humaines du Canada.

LeVasseur, L. (2008). Enseignement des disciplines scolaires et construction du sujet dans l'école québécoise. Éducation et francophonie, 36(2), 140-155.

LeVasseur, L. (2008-2012). La contribution de l'enseignement de l'histoire à la constitution de la culture commune. Fonds québécois de recherche sur la société et la culture.

LeVasseur, L. (2010). Individu, société et éducation (1960-2000). In M. Mellouki (dir.), Promesses et ratés de la réforme de l'éducation au Québec (pp. 51-73). Québec: Presses de l'Université Laval.

LeVasseur, L. (2012a). L'enseignement des matières scolaires et la transformation des modes de socialisation. In Y. Lenoir \& F. Tupin (dir.), Les pratiques enseignantes entre instruire et socialiser. Regards internationaux (pp 233-253). Québec : Presses de l'Université Laval.

LeVasseur, L. (2012b). L'école québécoise et la culture scolaire : développement intégral de l'enfant, développement cognitif de l'élève et contextes éducatifs. Phronesis, 1(4), 71-83.

LeVasseur, L. (2013). L'enseignement culturel de la religion au Québec et l'édification de la modernité. Revue d'éducation internationale de Sèvres, 63, 123-132.

LeVasseur, L., S. Moisan, \& Cardin, J.-F. (2013). Les enseignants d'histoire et le programme d'Histoire et d'éducation : de la transmission de la mémoire à une citoyenneté « subjective » et ouverte. Phronesis, 2(2-3), 77-86.

LeVasseur, L., \& J.-F. Cardin. (2012). The "social frameworks" of teaching high school history : teaching as part of the modernization of Québec society. Journal of Social Science Education, 11(4), 78-95. 
LeVasseur, L., \& Cardin, J.-F. (2013). L'enseignement de l'histoire au secondaire : du récit sur la nation au récit sur la modernité. Phronesis, 2(2-3), 63-76.

LeVasseur L., \& Tardif, M. (à paraître). La socialisation commune des élèves prise entre le pluralisme normatif et la différenciation statutaire des agents scolaires. Dossiers de l'éducation. Revue internationale des sciences de l'éducation.

LeVasseur, L., \& Tardif, M. (2005). L'essor du travail technique en milieu scolaire et son incidence sur l'organisation du travail. Recherches sociographiques, 46(1), 97-118.

Lyotard, J.-F. (1979). La condition postmoderne. Paris : Éditions de Minuit.

Martuccelli, D., \& de Singly, F. (2009). Les sociologies de l'individu. Paris : Armand Colin.

Ministère de l'Éducation du Québec. (1997), L'école, tout un programme. Énoncé de politique éducative. Québec : Gouvernement du Québec.

Simard, D. (2013). Carl Rogers et la pédagogie ouverte. In C. Gauthier \& M. Tardif (dir.), La pédagogie. Théories et pratiques de l'Antiquité à nos jours ( $3^{\mathrm{e}}$ édition), (pp. 156-171). Montréal : Gaëtan Morin Éditeur.

Tardif, M., \& LeVasseur, L. (2010). La division du travail éducatif. Paris : Presses universitaires de France.

Taylor, C. (1992). Grandeur et misère de la modernité, traduit de l'anglais (1991). Montréal : Bellarmin.

Testanière, J. (1967). Chahut traditionnel et chahut anomique dans l'enseignement du second degré. Revue française de sociologie, 8(hors-série), 17-33.

\section{NOTES}

1. Voir la partie 3 méthodologique et la note 6 pour l'identification des sujets de la recherche.

2. Recherche financée respectivement par le Conseil de recherche en sciences humaines du Canada (LeVasseur, 2007-2010), par le Fond québécois de recherche sur la société et la culture (LeVasseur, 2008-2012) et par la Bourse de développement de la recherche de la Faculté des sciences de l'éducation de l'Université Laval (LeVasseur, 2007-2008).

3. Dans un article subséquent (LeVasseur \& Tardif, à paraître), nous montrerons que de telles tensions peuvent saper les efforts, les énergies, les actions des uns et des autres et compromettre, de ce fait, l'encadrement des élèves au comportement difficile dans les établissements. Autrement dit, que la division du travail scolaire, la parcellisation des tâches et des rôles dans laquelle les établissements d'Amérique du Nord sont engagés n'est pas nécessairement garante de collaboration.

4. Pour des analyses portant sur la «subjectivation de la culture scolaire » dans différents programmes d'enseignement ou matières scolaires au Québec, voir: LeVasseur, 2013, 2012a, 2012b, 2008; LeVasseur et Cardin, 2013, 2012; LeVasseur, Moisan et Cardin, 2013.

5. Avant les années 1960, les francophones de la province du Québec se désignaient comme des Canadiens français. Après les années 1960, avec l'éveil d'un sentiment nationaliste moderne, plusieurs d'entre eux se sont désignés comme d'abord comme des Québécois.

6. Les sujets de nos recherches ont été codés préalablement à la rédaction de cet article. Ces codes ne correspondent pas à l'ordre dans lequel nous les citons. Notons que, dans cette section 4.1 , tous les extraits cités proviennent d'entretiens réalisés avec des enseignants d'histoire et de religion du secondaire. 
7. Dans cette section, nous indiquons l'ordre d'enseignement, primaire ou secondaire, dans lequel travaillent les enseignants et les TES.

8. Nous donnerons, dans un article à venir portant sur ce que nous appelons le pluralisme institutionnel, de nombreux exemples de l'absence de coordination de l'action entre agents scolaires intervenant auprès des élèves ayant des difficultés de comportement (LeVasseur \& Tardif, à paraître).

\section{RÉSUMÉS}

Depuis le début des années 1990, de nouveaux agents scolaires techniques et paratechniques, à côté des enseignants, du personnel administratif et des professionnels (psychologues, psychoéducateurs, orthopédagogues, orthophonistes), ont fait une entrée très importante, sur le plan numérique, dans les écoles primaires et secondaires au Québec. Parmi eux, les techniciens en éducation spécialisée exercent des tâches éducatives qui visent à soutenir l'élève sur les plans scolaire, social et psychologique, ce qui montre que l'école assume, en plus d'une mission d'instruction, une importante mission de socialisation. Comment, au sein des établissements, ces techniciens en éducation spécialisée se coordonnent-ils avec les enseignants sur le plan de la socialisation des élèves, qui est leur œuvre commune ? À bien des égards, comme le montre notre recherche en sociologie du travail éducatif, les techniciens en éducation spécialisée et les enseignants tiennent des discours analogues sur la socialisation des élèves, l'autonomie morale de la personne et la construction du sujet. Cependant, dépendamment du lieu institutionnel d'où ils parlent, de la fonction exercée, des élèves dont il est question et de la nature des problèmes de ceux-ci, les enseignants et ces agents techniques n'adhérent pas nécessairement aux mêmes modèles de socialisation et ne s'entendent pas quant à la priorité à donner soit à l'ordre scolaire, soit à l'autonomie du sujet, ce que nous illustrerons grâce à des données tirées de nos diverses recherches sur les modes de socialisation en milieu scolaire.

Since the early 1990s, the number of new education support personnel in Quebec's primary and secondary schools has increased considerably, complementing the teaching, administrative and professional staff (psychologists, psychoeducators, resource teachers and speech therapists). Special education aides are among the new education support personnel; they perform tasks designed to give students educational, social and psychological support, demonstrating that the mission of the school is to socialize, as well as instruct. In schools, how do special education aides coordinate with teachers in achieving their common goal of socializing students? In many respects, as shown by our research on the sociology of educational work, the discourse of teachers and special education aides is similar with respect to student socialization, moral autonomy and the construction of the subject. However, depending on the institutional ground from which they speak, the duties they perform, the students involved and the nature of their problems, teachers and special education aides do not necessarily adhere to the same models of socialization and do not agree on whether to prioritize academic aspects or the subject's autonomy, as we will show using data drawn from our studies of models of socialization in schools. 
INDEX

Mots-clés : division du travail, travail enseignant et technique, subjectivation, ordre scolaire, socio-affectivité

Keywords : division of work, teacher and technical work, subjectification, school order, socioaffectivity

\section{AUTEUR}

\section{LOUIS LEVASSEUR}

Université Laval et Centre de recherche interuniversitaire sur la formation et la profession enseignante (CRIFPE) ; Faculté des sciences de l'éducation 